\title{
Equilibrium analysis for an epidemic model with a reservoir for infection
}

\author{
Istvan Lauko, Gabriella Pinter and Rachel Elizabeth TeWinkel \\ University of Wisconsin-Milwaukee, Milwaukee, WI, USA
}

\begin{abstract}
We consider a system of non-linear differential equations describing the spread of an epidemic in two interacting populations. The model assumes that the epidemic spreads within the first population, which in turn acts as a reservoir of infection for the second population. We explore the conditions under which the epidemic is endemic in both populations and discuss the global asymptotic stability of the endemic equilibrium using a Lyapunov function and results established for asymptotically autonomous systems. We discuss monkeypox as an example of an emerging disease that can be modelled in this way and present some numerical results representing the model and its extensions.
\end{abstract}

\section{ARTICLE HISTORY}

Received 25 May 2018

Accepted 16 November 2018

\section{KEYWORDS}

Monkeypox; Lyapunov function; epidemic model; systems of ODEs; global stability

\section{Introduction}

In this paper we consider an epidemic model motivated by monkeypox, an emerging disease that has become more prevalent recently in several areas of Africa (Bhunu \& Mushayabasa, 2011; Bhunu, Mushayabasa, \& Hyman, 2012; Damon, 2011; Hammarlund et al., 2005; Hutin et al., 2001; Kantele, Chickering, Vapalahti, \& Rimoin, 2016; Levine, Townsend, Carroll, Damon, \& Reynolds, 2007; McCollum \& Damon, 2014; The Center for Food Security and Public Health, 2013). It is believed that the noticeable increase in monkeypox (Nolen et al., 2016) is linked to the decrease in herd immunity to smallpox (Hutin et al., 2001; Levine et al., 2007; Lloyd-Smith et al., 2009; McCollum \& Damon, 2014) due to the phasing out of smallpox vaccinations (Nolen et al., 2016; Rimoin \& Graham, 2011; Rimoin et al., 2007, 2010). Hosts of the monkeypox virus include prairie dogs, tree squirrels, chimpanzees, and baboons, but the complete list of pathogen hosts is not known (Centers for Disease Control and Prevention, 2015; Reynolds et al., 2013; The Center for Food Security and Public Health, 2013; World Health Organization, 2016). Monkeypox infects both humans and animals, and is generally considered impossible to eradicate (Damon, 2011; Kantele et al., 2016; McCollum \& Damon, 2014; Reynolds et al., 2013; Rimoin \& Graham, 2011). Humans become infected with the monkeypox virus when they come into direct contact with infected animals or other humans (Centers for Disease Control and Prevention, 2015; Hammarlund et al., 2005; Jezek, Arita, 
Mutombo, \& Szczeniowski, 1986; Jezek, Grab, Szczeniowski, Paluku, \& Mutombo, 1988; The Center for Food Security and Public Health, 2013; Weaver \& Isaacs, 2009; World Health Organization, 2016). Since human-animal cross-infection usually occurs when humans hunt and eat animals, we can assume that animals do not become infected via contact with the human population, but that animals can infect humans (Reynolds et al., 2013). This creates an asymmetric disease transmission between the animal and human populations, and we can effectively treat the animal population as a reservoir for the disease within which disease dynamics are independent from the disease's course in the human population. Monkeypox was explicitly modelled by Bhunu and Mushayabasa and Bhunu, Mushayabasa and Hyman with traditional SIR models in both the animal and human population, and with standard incidence for disease transmission (Bhunu \& Mushayabasa, 2011; Bhunu et al., 2012). However, the analysis presented in Bhunu and Mushayabasa (2011) and Bhunu et al. (2012) is not complete, and here we present an alternative full equilibrium analysis for the first model by Bhunu and Mushayabasa, and establish global stability of the endemic equilibrium in both populations under suitable conditions on the parameters (Bhunu \& Mushayabasa, 2011). We utilize the theory developed by Markus (1956) and later by Thieme and Castillo-Chavez (Castillo-Chavez \& Thieme, 1994; Thieme, 1992) for asymptotically autonomous systems together with the techniques of identifying suitable Lyapunov functions for SIR models with standard incidence described by Vargas-De-León (2011).

We note that these ideas can be readily generalized for multiple reservoir (and human) populations, and are more widely applicable than the special case of monkeypox. In particular, birds can harbour avian flu viruses that can adapt to and spread in human populations (Centers for Disease Control and Prevention, 2017a), bats are a reservoir for rabies (Gilbert et al., 2015), and ebola viruses are also thought to have a natural animal reservoir (Centers for Disease Control and Prevention, 2017b). The model considered in this paper would suggest that once the disease becomes endemic in the reservoir population, endemic disease in the human population is inevitable. Thus it could be suggested that an effective control measure would be the mass culling of animals that harbour the disease causing pathogens to prevent the establishment of an endemic disease in the animal population. While this strategy is widely used for controlling avian flu within domestic bird populations (Centers for Disease Control and Prevention, 2017a), it is not practical or even possible for the cases of monkeypox, rabies, or ebola within wild populations. The prediction of endemic levels of the disease in the human population (while it may be a good approximation at times) are not wholly realistic, due to fluctuations in incidence of monkeypox in the human population (Nolen et al., 2016).

\section{Description of the model}

We consider two populations, denoted by $A$ and $H$, in which a disease spreads. Both populations are divided into susceptible, infectious and recovered individuals, denoted by $S_{a}, I_{a}$ and $R_{a}$, and $S_{h}, I_{h}$ and $R_{h}$, respectively. The total number of individuals in each population is given as $N_{a}(t)=S_{a}(t)+I_{a}(t)+R_{a}(t)$ and $N_{h}(t)=S_{h}(t)+I_{h}(t)+R_{h}(t)$. Susceptible individuals in $A$ are recruited through migration and birth at the rate $\Lambda_{a}$ and susceptible individuals in $H$ are recruited at a rate of $\Lambda_{h}$ (Bhunu \& Mushayabasa, 2011). Let $d_{a}, d_{h}$ be the death rates by the disease for $A$ and $H$, respectively, $\mu_{a}, \mu_{h}$ be the natural death 
rates for $A$ and $H$, respectively, and $\rho_{a}, \rho_{h}$ be the recovery rates with permanent immunity for $A$ and $H$, respectively. It is assumed that no one in population $H$ can infect any individual in population $A$, while individuals in $A$ can infect those in $H$ on suitable contact. Although this model can be used to describe epidemics of different diseases, in the case of monkeypox population $A$ is the animal population and $H$ represents the human population. Disease transmission is modelled using standard incidence, assuming a constant (density-independent) contact rate both within and across the two populations resulting in infection rates

$$
f_{a}\left(S_{a}, I_{a}, R_{a}\right)=\frac{\beta_{a_{1}} I_{a}}{N_{a}} S_{a}, \quad \text { and } \quad f_{h}\left(S_{a}, I_{a}, R_{a}, S_{h}, I_{h}, R_{h}\right)=\left(\frac{\beta_{a_{2}} I_{a}}{N_{a}}+\frac{\beta_{h} I_{h}}{N_{h}}\right) S_{h},
$$

where $\beta_{a_{1}}, \beta_{a_{2}}$ and $\beta_{h}$ are the effective contact rates in population $A$, between populations $A$ and $H$, and in population $H$, respectively. We assume that $\Lambda_{a}, \Lambda_{h}, \mu_{a}, \mu_{h}, \rho_{a}, \rho_{h}$ are positive, while $d_{a}, d_{h}, \beta_{a_{1}}, \beta_{a_{2}}$ and $\beta_{h}$ are non-negative parameters. Thus we have the following system of non-linear differential equations (1a)-(1f) (Bhunu \& Mushayabasa, 2011)

$$
\begin{gathered}
\frac{\mathrm{d} S_{a}}{\mathrm{~d} t}=\Lambda_{a}-\mu_{a} S_{a}-\frac{\beta_{a_{1}} I_{a}}{N_{a}} S_{a}, \\
\frac{\mathrm{d} I_{a}}{\mathrm{~d} t}=\frac{\beta_{a_{1}} I_{a}}{N_{a}} S_{a}-\left(\mu_{a}+\rho_{a}+d_{a}\right) I_{a}, \\
\frac{\mathrm{d} R_{a}}{\mathrm{~d} t}=\rho_{a} I_{a}-\mu_{a} R_{a}, \\
\frac{\mathrm{d} S_{h}}{\mathrm{~d} t}=\Lambda_{h}-\mu_{h} S_{h}-\left(\frac{\beta_{a_{2}} I_{a}}{N_{a}}+\frac{\beta_{h} I_{h}}{N_{h}}\right) S_{h}, \\
\frac{\left(\frac{\beta_{a_{2}} I_{a}}{N_{a}}+\frac{\beta_{h} I_{h}}{N_{h}}\right) S_{h}-\left(\mu_{h}+\rho_{h}+d_{h}\right) I_{h},}{\frac{\mathrm{d} R_{h}}{\mathrm{~d} t}=\rho_{h} I_{h}-\mu_{h} R_{h} .}
\end{gathered}
$$

Since

$$
N_{a}^{\prime}=\Lambda_{a}-\mu_{a} N_{a}-d_{a} I_{a} \quad \text { and } \quad N_{h}^{\prime}=\Lambda_{h}-\mu_{h} N_{h}-d_{h} I_{h},
$$

the set $\Omega=\Omega_{a} \times \Omega_{h}$, where

$$
\Omega_{a}=\left\{\left(S_{a}, I_{a}, R_{a}\right) \in \mathbb{R}_{+}^{3}: S_{a} \geq 0, I_{a} \geq 0, R_{a} \geq 0, S_{a}+I_{a}+R_{a} \leq \frac{\Lambda_{a}}{\mu_{a}}\right\}
$$

and

$$
\Omega_{h}=\left\{\left(S_{h}, I_{h}, R_{h}\right) \in \mathbb{R}_{+}^{3}: S_{h} \geq 0, I_{h} \geq 0, R_{h} \geq 0, S_{h}+I_{h}+R_{h} \leq \frac{\Lambda_{h}}{\mu_{h}}\right\},
$$

is positively invariant under the dynamics of (1a)-(1f), and solutions with initial conditions in $\Omega$ exist globally. 


\section{Equilibrium analysis}

System (1a)-(1f) has several different types of potential equilibria: disease-free, endemic disease in only one of the populations and endemic disease in both populations. Using the method of van der Driessche and Watmough (Bhunu \& Mushayabasa, 2011; CastilloChavez, Feng, \& Huang, 2001; van den Driessche \& Watmough, 2002), the basic reproduction numbers of the model are $\mathcal{R}_{0_{a}}$ and $\mathcal{R}_{0_{h}}$, where

$$
\mathcal{R}_{0_{a}}=\frac{\beta_{a_{1}}}{\mu_{a}+\rho_{a}+d_{a}} \quad \text { and } \quad \mathcal{R}_{0_{h}}=\frac{\beta_{h}}{\mu_{h}+\rho_{h}+d_{h}} .
$$

In the following we address the feasibility and stability properties of each of the different equilibria of the system (1a)-(1f). Our analysis relies heavily on the results of Vargas-DeLeón given below (Vargas-De-León, 2011).

Consider the dynamics of the disease in the reservoir population $A$ given by (1a)-(1c). The system has a disease-free equilibrium $E_{0_{a}}=\left(\Lambda_{a} / \mu_{a}, 0,0\right)$ and a unique endemic equilibrium $E_{a}^{*}$ with coordinates

$$
\begin{gathered}
S_{a}^{*}=\frac{\Lambda_{a}\left(\rho_{a}+\mu_{a}\right)}{\mu_{a}\left(\rho_{a}+\mu_{a}\right) \mathcal{R}_{0_{a}}+d_{a} \mu_{a}\left(\mathcal{R}_{0_{a}}-1\right)}=\frac{\Lambda_{a}}{\mu_{a}}-\frac{\mu_{a}+\rho_{a}+d_{a}}{\mu_{a}} I_{a}^{*}, \\
I_{a}^{*}=\frac{\Lambda_{a} \mu_{a}\left(\mathcal{R}_{0_{a}}-1\right)}{\mu_{a}\left(\rho_{a}+\mu_{a}\right) \mathcal{R}_{0_{a}}+d_{a} \mu_{a}\left(\mathcal{R}_{0_{a}}-1\right)}=\frac{\Lambda_{a}\left(\beta_{a_{1}}-\left(\mu_{a}+\rho_{a}+d_{a}\right)\right)}{\left(\beta_{a_{1}}-d_{a}\right)\left(\mu_{a}+\rho_{a}+d_{a}\right)}, \\
R_{a}^{*}=\frac{\Lambda_{a} \rho_{a}\left(\mathcal{R}_{0_{a}}-1\right)}{\mu_{a}\left(\rho_{a}+\mu_{a}\right) \mathcal{R}_{0_{a}}+d_{a} \mu_{a}\left(\mathcal{R}_{0_{a}}-1\right)}=\frac{\rho_{a}}{\mu_{a}} I_{a}^{*} .
\end{gathered}
$$

We note that $I_{a}^{*}>0$ whenever $\mathcal{R}_{0_{a}}>1$ or $\beta_{a_{1}}<d_{a}$, but in the latter case the resulting $S_{a}^{*}$ is negative. Thus the endemic equilibrium is feasible, that is, $E_{a}^{*} \in \Omega_{a}$, if and only if $\mathcal{R}_{0_{a}}>1$. The following theorems are proved by Vargas-De-León by the construction of appropriate Lyapunov functions (Vargas-De-León, 2011).

Theorem 3.1: If $\mathcal{R}_{0_{a}} \leq 1$, then the disease-free equilibrium $E_{0_{a}}$ of (1a)-(1c) is globally asymptotically stable in $\Omega_{a}$.

Theorem 3.2: Assume that $\mu_{a}>d_{a}$ and $\mathcal{R}_{0_{a}}>1$, then the unique endemic equilibrium $E_{a}^{*}$ of (1a)-(1c) is globally asymptotically stable in the interior of $\Omega_{a}$.

Once we know the dynamics of the disease in population $A$ from the theorems above, we can consider how this affects disease propagation in population $H$. Here our analysis diverges from what was done previously in Bhunu and Mushayabasa (2011). Individuals in $H$ can get infected by contact with infectious individuals in population $A$ or $H$. However, we know that if $\mathcal{R}_{0_{a}} \leq 1$, then $I_{a}(t) \rightarrow 0, N_{a}(t) \rightarrow \Lambda_{a} / \mu_{a}$ as $t \rightarrow \infty$, while if $\mathcal{R}_{0_{a}}>1$ and $\mu_{a}>d_{a}$, then $I_{a}(t) \rightarrow I_{a}^{*}>0, N_{a}(t) \rightarrow N_{a}^{*}=S_{a}^{*}+I_{a}^{*}+R_{a}^{*}$ as $t \rightarrow \infty$. Thus we can 
think of (1d)-(1f) as a non-autonomous system

$$
\begin{gathered}
\frac{\mathrm{d} S_{h}}{\mathrm{~d} t}=\Lambda_{h}-\mu_{h} S_{h}-\left(\beta_{a_{2}} g(t)+\frac{\beta_{h} I_{h}}{N_{h}}\right) S_{h}, \\
\frac{\mathrm{d} I_{h}}{\mathrm{~d} t}=\left(\beta_{a_{2}} g(t)+\frac{\beta_{h} I_{h}}{N_{h}}\right) S_{h}-\left(\mu_{h}+\rho_{h}+d_{h}\right) I_{h}, \\
\frac{\mathrm{d} R_{h}}{\mathrm{~d} t}=\rho_{h} I_{h}-\mu_{h} R_{h},
\end{gathered}
$$

where $g(t):=I_{a}(t) / N_{a}(t)$. By Theorems 3.1 and 3.2, we have that

$$
\lim _{t \rightarrow \infty} g(t)=\frac{\lim _{t \rightarrow \infty} I_{a}(t)}{\lim _{t \rightarrow \infty} N_{a}(t)}=\frac{I_{a}^{e}}{N_{a}^{e}},
$$

where the limits $I_{a}^{e}$ and $N_{a}^{e}$ depend on $\mathcal{R}_{0_{a}}$ and the corresponding parameters. That is, $I_{a}^{e}=0$ when $\mathcal{R}_{0_{a}} \leq 1$ and $I_{a}^{e}=I_{a}^{*}$ when $\mathcal{R}_{0_{a}}>1$. This makes (1d)-(1f) an asymptotically autonomous system with limit system

$$
\begin{gathered}
\frac{\mathrm{d} S_{h}}{\mathrm{~d} t}=\Lambda_{h}-\mu_{h} S_{h}-\left(\beta_{a_{2}} \frac{I_{a}^{e}}{N_{a}^{e}}+\frac{\beta_{h} I_{h}}{N_{h}}\right) S_{h}, \\
\frac{\mathrm{d} I_{h}}{\mathrm{~d} t}=\left(\beta_{a_{2}} \frac{I_{a}^{e}}{N_{a}^{e}}+\frac{\beta_{h} I_{h}}{N_{h}}\right) S_{h}-\left(\mu_{h}+\rho_{h}+d_{h}\right) I_{h}, \\
\frac{\mathrm{d} R_{h}}{\mathrm{~d} t}=\rho_{h} I_{h}-\mu_{h} R_{h},
\end{gathered}
$$

and we can use the theory developed for such systems (Castillo-Chavez \& Thieme, 1994; Thieme, 1992) to address the stability properties of our model in all possible cases. In particular, we repeatedly make use of the following corollary (Thieme, 1992) applied to our systems:

Corollary 3.3: If solutions of the system (3a)-(3c) are bounded, and the equilibrium $E$ of the limit system (4a)-(4c) is globally asymptotically stable, then any solution $\left(S_{h}(t), I_{h}(t), R_{h}(t)\right)$ of the system (3a)-(3c) satisfies $\left(S_{h}(t), I_{h}(t), R_{h}(t)\right) \rightarrow E$ as $t \rightarrow \infty$.

\subsection{Endemic equilibrium in both populations}

We start with the following claim:

Proposition 3.4: Assume that $\mathcal{R}_{0_{a}}>1, \beta_{a_{2}}>0$ and $\mu_{a}>d_{a}$, so the disease in the reservoir population tends to the endemic equilibrium $E_{a}^{*}$. Then (1d)-(1f) has a unique endemic equilibrium $E_{h}^{*} \in \Omega_{h}$, and $\mathcal{E}_{1}=\left(E_{a}^{*}, E_{h}^{*}\right)$ is the unique endemic equilibrium of (1a)-(1f) in $\Omega$. 
Proof: To calculate $E_{h}^{*}=\left(S_{h}^{*}, I_{h}^{*}, R_{h}^{*}\right)$, we follow the steps in (Bhunu \& Mushayabasa, 2011), and set the right sides of Equations (1d)-(1f) equal to zero to obtain

$$
\begin{gathered}
\Lambda_{h}=\left(\mu_{h}+\frac{\beta_{a_{2}} I_{a}^{*}}{N_{a}^{*}}+\frac{\beta_{h} I_{h}^{*}}{N_{h}^{*}}\right) S_{h}^{*}, \\
\left(\frac{\beta_{a_{2}} I_{a}^{*}}{N_{a}^{*}}+\frac{\beta_{h} I_{h}^{*}}{N_{h}^{*}}\right) S_{h}^{*}=\left(\mu_{h}+\rho_{h}+d_{h}\right) I_{h}^{*}, \\
\rho_{h} I_{h}^{*}=\mu_{h} R_{h}^{*} .
\end{gathered}
$$

In Bhunu and Mushayabasa (2011), the unique endemic equilibrium was found subject to $\beta_{h}>d_{h}\left(1+\beta_{a_{2}} I_{a}^{*} / \mu_{h} N_{a}^{*}\right)$. Instead, we show the existence of the unique endemic equilibrium using a method similar to Afassinou, Chirove, and Govinder (2017) that holds regardless of the stated inequality.

For simplification, define

$$
\begin{aligned}
& \lambda_{a}^{*}=\frac{\beta_{a_{2}} I_{a}^{*}}{N_{a}^{*}}, \quad m_{h}=\mu_{h}+\rho_{h}+d_{h}, \quad \lambda_{h}^{*}=\frac{\beta_{h} I_{h}^{*}}{N_{h}^{*}}, \\
& a=\frac{\Lambda_{h} \lambda_{a}^{*}}{m_{h}}, \quad b=\frac{\Lambda_{h}}{m_{h}}, \quad c=\frac{\rho_{h} a}{\mu_{h}}, \quad d=\frac{\rho_{h} b}{\mu_{h}}, \quad e=\Lambda_{h}+a+c, \quad f=b+d .
\end{aligned}
$$

Using (5),

$$
S_{h}^{*}=\frac{\Lambda_{h}}{\mu_{h}+\lambda_{a}^{*}+\lambda_{h}^{*}}
$$

From (6) and using (8),

$$
I_{h}^{*}=\frac{S_{h}^{*}\left(\lambda_{a}^{*}+\lambda_{h}^{*}\right)}{m_{h}}=\left(\frac{\Lambda_{h}}{\mu_{h}+\lambda_{a}^{*}+\lambda_{h}^{*}}\right)\left(\frac{\lambda_{a}^{*}+\lambda_{h}^{*}}{m_{h}}\right)=\frac{a+b \lambda_{h}^{*}}{\mu_{h}+\lambda_{a}^{*}+\lambda_{h}^{*}} .
$$

Clearly, by (7) and (9),

$$
R_{h}^{*}=\frac{\rho_{h}}{\mu_{h}} I_{h}^{*}=\left(\frac{a+b \lambda_{h}^{*}}{\mu_{h}+\lambda_{a}^{*}+\lambda_{h}^{*}}\right)\left(\frac{\rho_{h}}{\mu_{h}}\right)=\frac{c+d \lambda_{h}^{*}}{\mu_{h}+\lambda_{a}^{*}+\lambda_{h}^{*}} .
$$

Then it follows that

$$
\frac{I_{h}^{*}}{N_{h}^{*}}=\left(\frac{a+b \lambda_{h}^{*}}{\mu_{h}+\lambda_{a}^{*}+\lambda_{h}^{*}}\right)\left(\frac{\mu_{h}+\lambda_{a}^{*}+\lambda_{h}^{*}}{\Lambda_{h}+a+b \lambda_{h}^{*}+c+d \lambda_{h}^{*}}\right)=\frac{a+b \lambda_{h}^{*}}{e+f \lambda_{h}^{*}}
$$

so that

$$
\lambda_{h}^{*}=\frac{\beta_{h}\left(a+b \lambda_{h}^{*}\right)}{e+f \lambda_{h}^{*}}
$$

Rearranging (11), we obtain

$$
f \lambda_{h}^{* 2}+\lambda_{h}^{*}\left(e-\beta_{h} b\right)-\beta_{h} a=0 .
$$

The above quadratic equation in $\lambda_{h}^{*}$ has one positive root. Now (8), (9) and (10) imply that the corresponding $E_{h}^{*}=\left(S_{h}^{*}, I_{h}^{*}, R_{h}^{*}\right)$ is feasible. 
Consider now the endemic equilibrium $\mathcal{E}_{1}=\left(E_{a}^{*}, E_{h}^{*}\right) \in \Omega$. We prove the following theorem.

Theorem 3.5: If $\mathcal{R}_{0_{a}}>1, \mu_{a}>d_{a}, \beta_{a_{2}}>0$ and $\mu_{h}>d_{h}$ then the unique endemic equilibrium $\mathcal{E}_{1}=\left(E_{a}^{*}, E_{h}^{*}\right)$ of (1a)-(1f) is globally asymptotically stable in the interior of $\Omega$.

Proof: Since $\mathcal{R}_{0_{a}}>1$ and $\mu_{a}>d_{a}$ Theorem 3.2 implies that $E_{a}^{*}$ is a globally asymptotically stable equilibrium of (1a)-(1c) in the interior of $\Omega_{a}$. Using this result, and the assumption that $\beta_{a_{2}}>0$ we have that the system

$$
\begin{aligned}
\frac{\mathrm{d} S_{h}}{\mathrm{~d} t} & =\Lambda_{h}-\mu_{h} S_{h}-\left(\beta_{a_{2}} \frac{I_{a}^{*}}{N_{a}^{*}}+\frac{\beta_{h} I_{h}}{N_{h}}\right) S_{h}, \\
\frac{\mathrm{d} I_{h}}{\mathrm{~d} t} & =\left(\beta_{a_{2}} \frac{I_{a}^{*}}{N_{a}^{*}}+\frac{\beta_{h} I_{h}}{N_{h}}\right) S_{h}-\left(\mu_{h}+\rho_{h}+d_{h}\right) I_{h}, \\
\frac{\mathrm{d} R_{h}}{\mathrm{~d} t} & =\rho_{h} I_{h}-\mu_{h} R_{h}
\end{aligned}
$$

is the asymptotic limit of system (1d)-(1f), and $E_{h}^{*}$ is its unique equilibrium. We claim that $E_{h}^{*}$ is a globally asymptotically stable equilibrium of $(4 \mathrm{a})-(4 \mathrm{c})$.

Consider the function $L:\left\{\left(S_{h}, I_{h}, R_{h}\right) \in \Omega_{h}: S_{h}>0, I_{h}>0, R_{h}>0\right\} \rightarrow \mathbb{R}$

$$
\begin{aligned}
L= & N_{h}-N_{h}^{*}-N_{h}^{*} \ln \left(\frac{N_{h}}{N_{h}^{*}}\right)+\frac{N_{h}^{*}\left(d_{h}+2 \mu_{h}\right)}{\beta_{h}\left(I_{h}^{*}+R_{h}^{*}\right)}\left[I_{h}-I_{h}^{*}-I_{h}^{*} \ln \left(\frac{I_{h}}{I_{h}^{*}}\right)\right] \\
& +\frac{\left(d_{h}+2 \mu_{h}\right)}{2 \rho_{h}}\left(1+\frac{S_{h}^{*}}{I_{h}^{*}+R_{h}^{*}}\right)\left(\frac{\left(R_{h}-R_{h}^{*}\right)^{2}}{N_{h}}\right)+D\left(N_{h}-N_{h}^{*}\right)^{2}+E\left(R_{h}-R_{h}^{*}\right)^{2},
\end{aligned}
$$

where $D, E>0$ are yet to be determined. $L \in C^{1}\left(\Omega_{h}\right), L\left(S_{h}^{*}, I_{h}^{*}, R_{h}^{*}\right)=0$ and $L$ is positive semi-definite. Calculating the derivative of $L$ along solutions of (4a) $-(4 \mathrm{c})$ and using the relationships

$$
\begin{aligned}
& \Lambda_{h}=\left(\mu_{h}+\frac{\beta_{a_{2}} I_{a}^{*}}{N_{a}^{*}}+\frac{\beta_{h} I_{h}^{*}}{N_{h}^{*}}\right) S_{h}^{*}, \quad \frac{\beta_{a_{2}} I_{a}^{*}}{N_{a}^{*}}+\frac{\beta_{h} I_{h}^{*}}{N_{h}^{*}} S_{h}^{*}=\left(\mu_{h}+\rho_{h}+d_{h}\right) I_{h}^{*}, \\
& \rho_{h} I_{h}^{*}=\mu_{h} R_{h}^{*},
\end{aligned}
$$

we obtain

$$
\begin{aligned}
L^{\prime}= & \left(\mu_{h}\left(S_{h}^{*}+I_{h}^{*}+R_{h}^{*}\right)+d_{h} I_{h}^{*}-\mu_{h}\left(S_{h}+I_{h}+R_{h}\right)-d_{h} I_{h}\right)\left(\frac{N_{h}-N_{h}^{*}}{N_{h}}\right) \\
& +\frac{N_{h}^{*}\left(d_{h}+2 \mu_{h}\right)}{\beta_{h}\left(I_{h}^{*}+R_{h}^{*}\right)}\left[\frac{\beta_{a_{2}} I_{a}^{*} S_{h} I_{h}^{*}\left(I_{h}-I_{h}^{*}\right)}{N_{a}^{*} I_{h} I_{h}^{*}}-\frac{\beta_{a_{2}} I_{a}^{*} S_{h}^{*} I_{h}\left(I_{h}-I_{h}^{*}\right)}{N_{a}^{*} I_{h} I_{h}^{*}}\right. \\
& \left.+\frac{\beta_{h} S_{h} N_{h}^{*}\left(I_{h}-I_{h}^{*}\right)}{N_{h}^{*} N_{h}}-\frac{\beta_{h} S_{h}^{*} N_{h}\left(I_{h}-I_{h}^{*}\right)}{N_{h}^{*} N_{h}}\right]
\end{aligned}
$$




$$
\begin{aligned}
& +\frac{\left(d_{h}+2 \mu_{h}\right)}{2 \rho_{h}}\left(1+\frac{S_{h}^{*}}{I_{h}^{*}+R_{h}^{*}}\right)\left(\frac{2\left(\rho_{h}\left(I_{h}-I_{h}^{*}\right)-\mu_{h}\left(R_{h}-R_{h}^{*}\right)\right)\left(R_{h}-R_{h}^{*}\right)}{N_{h}}\right. \\
& \left.-\frac{N_{h}^{\prime}\left(R_{h}-R_{h}^{*}\right)^{2}}{N_{h}^{2}}\right)+2 D N_{h}^{\prime}\left(N_{h}-N_{h}^{*}\right) \\
& +2 E\left(\rho_{h} I_{h}-\mu_{h} R_{h}-\rho_{h} I_{h}^{*}+\mu_{h} R_{h}^{*}\right)\left(R_{h}-R_{h}^{*}\right) .
\end{aligned}
$$

Since

$$
\begin{aligned}
& \beta_{h} S_{h} N_{h}^{*}\left(I_{h}-I_{h}^{*}\right)-\beta_{h} S_{h}^{*} N_{h}\left(I_{h}-I_{h}^{*}\right)=\beta_{h}\left(I_{h}-I_{h}^{*}\right)\left(S_{h} N_{h}^{*}-S_{h}^{*} N_{h}\right) \\
& =\beta_{h}\left(I_{h}-I_{h}^{*}\right)\left(\left(S_{h}-S_{h}^{*}\right)\left(I_{h}^{*}+R_{h}^{*}\right)-S_{h}^{*}\left(I_{h}-I_{h}^{*}\right)-S_{h}^{*}\left(R_{h}-R_{h}^{*}\right)\right)
\end{aligned}
$$

and

$$
\beta_{a_{2}} I_{a}^{*} S_{h} I_{h}^{*}\left(I_{h}-I_{h}^{*}\right)-\beta_{a_{2}} I_{a}^{*} S_{h}^{*} I_{h}\left(I_{h}-I_{h}^{*}\right)=\beta_{a_{2}} I_{a}^{*}\left(I_{h}-I_{h}^{*}\right)\left(I_{h}\left(S_{h}-S_{h}^{*}\right)-S_{h}\left(I_{h}-I_{h}^{*}\right)\right),
$$

we have

$$
\begin{aligned}
L^{\prime}= & \left(\mu_{h}\left(S_{h}^{*}+I_{h}^{*}+R_{h}^{*}\right)+d_{h} I_{h}^{*}-\mu_{h}\left(S_{h}+I_{h}+R_{h}\right)-d_{h} I_{h}\right)\left(\frac{N_{h}-N_{h}^{*}}{N_{h}}\right) \\
& +\frac{N_{h}^{*}\left(d_{h}+2 \mu_{h}\right)}{\beta_{h}\left(I_{h}^{*}+R_{h}^{*}\right)}\left[\frac{\beta_{a_{2}} I_{a}^{*}\left(I_{h}-I_{h}^{*}\right)\left(S_{h}-S_{h}^{*}\right)}{N_{a}^{*} I_{h}^{*}}-\frac{\beta_{a_{2}} I_{a}^{*} S_{h}\left(I_{h}-I_{h}^{*}\right)^{2}}{N_{a}^{*} I_{h} I_{h}^{*}}\right. \\
& +\frac{\beta_{h}\left(I_{h}-I_{h}^{*}\right)\left(S_{h}-S_{h}^{*}\right)\left(I_{h}^{*}+R_{h}^{*}\right)}{N_{h}^{*} N_{h}}-\frac{\beta_{h} S_{h}^{*}\left(I_{h}-I_{h}^{*}\right)^{2}}{N_{h}^{*} N_{h}} \\
& \left.-\frac{\beta_{h} S_{h}^{*}\left(I_{h}-I_{h}^{*}\right)\left(R_{h}-R_{h}^{*}\right)}{N_{h}^{*} N_{h}}\right] \\
& +\frac{\left(d_{h}+2 \mu_{h}\right)}{2 \rho_{h}}\left(1+\frac{S_{h}^{*}}{I_{h}^{*}+R_{h}^{*}}\right)\left(\frac{2 \rho_{h}\left(I_{h}-I_{h}^{*}\right)\left(R_{h}-R_{h}^{*}\right)}{N_{h}}\right. \\
& \left.-\frac{2 \mu_{h}\left(R_{h}-R_{h}^{*}\right)^{2}}{N_{h}}-\frac{N_{h}^{\prime}\left(R_{h}-R_{h}^{*}\right)^{2}}{N_{h}^{2}}\right) \\
& +2 D N_{h}^{\prime}\left(N_{h}-N_{h}^{*}\right)+2 E \rho_{h}\left(I_{h}-I_{h}^{*}\right)\left(R_{h}-R_{h}^{*}\right)-2 E \mu_{h}\left(R_{h}-R_{h}^{*}\right)^{2} .
\end{aligned}
$$

Notice that the first term in $L^{\prime}$ can be written as

$$
\begin{aligned}
& \left(\frac{\left(S_{h}-S_{h}^{*}\right)+\left(I_{h}-I_{h}^{*}\right)+\left(R_{h}-R_{h}^{*}\right)}{N_{h}}\right)\left(-\mu_{h}\left(S_{h}-S_{h}^{*}+I_{h}-I_{h}^{*}+R_{h}-R_{h}^{*}\right)\right. \\
& \left.\quad-d_{h}\left(I_{h}-I_{h}^{*}\right)\right)
\end{aligned}
$$




$$
\begin{aligned}
= & -\frac{\mu_{h}\left(\left(S_{h}-S_{h}^{*}\right)-\left(R_{h}-R_{h}^{*}\right)\right)^{2}}{N_{h}}-\frac{\left(d_{h}+\mu_{h}\right)\left(I_{h}-I_{h}^{*}\right)^{2}}{N_{h}} \\
- & \frac{\left(d_{h}+2 \mu_{h}\right)\left(S_{h}-S_{h}^{*}\right)\left(I_{h}-I_{h}^{*}\right)}{N_{h}} \\
& -\frac{\left(d_{h}+2 \mu_{h}\right)\left(I_{h}-I_{h}^{*}\right)\left(R_{h}-R_{h}^{*}\right)}{N_{h}},
\end{aligned}
$$

so we can write

$$
\begin{aligned}
L^{\prime}= & -\frac{\mu_{h}\left(\left(S_{h}-S_{h}^{*}\right)-\left(R_{h}-R_{h}^{*}\right)\right)^{2}}{N_{h}}-\frac{\left(d_{h}+\mu_{h}\right)\left(I_{h}-I_{h}^{*}\right)^{2}}{N_{h}} \\
& +\frac{N_{h}^{*}\left(d_{h}+2 \mu_{h}\right)}{\beta_{h}\left(I_{h}^{*}+R_{h}^{*}\right)}\left(\frac{\beta_{a_{2}} I_{a}^{*}\left(I_{h}-I_{h}^{*}\right)\left(S_{h}-S_{h}^{*}\right)}{N_{a}^{*} I_{h}^{*}}\right) \\
& -\left(\frac{N_{h}^{*}\left(d_{h}+2 \mu_{h}\right)}{\beta_{h}\left(I_{h}^{*}+R_{h}^{*}\right)}\right)\left(\frac{\beta_{a_{2}} I_{a}^{*} S_{h}\left(I_{h}-I_{h}^{*}\right)^{2}}{N_{a}^{*} I_{h} I_{h}^{*}}+\frac{\beta_{h} S_{h}^{*}\left(I_{h}-I_{h}^{*}\right)^{2}}{N_{h}^{*} N_{h}}\right) \\
& -\frac{\left(d_{h}+2 \mu_{h}\right)}{2 \rho_{h}}\left(1+\frac{S_{h}^{*}}{I_{h}^{*}+R_{h}^{*}}\right)\left(\frac{2 \mu_{h}\left(R_{h}-R_{h}^{*}\right)^{2}}{N_{h}}+\frac{N_{h}^{\prime}\left(R_{h}-R_{h}^{*}\right)^{2}}{N_{h}^{2}}\right) \\
& +2 D N_{h}^{\prime}\left(N_{h}-N_{h}^{*}\right)+2 E \rho_{h}\left(I_{h}-I_{h}^{*}\right)\left(R_{h}-R_{h}^{*}\right)-2 E \mu_{h}\left(R_{h}-R_{h}^{*}\right)^{2} .
\end{aligned}
$$

Now rearranging the terms as in (13), we obtain

$$
\begin{aligned}
L^{\prime}= & -\frac{\mu_{h}\left(\left(S_{h}-S_{h}^{*}\right)-\left(R_{h}-R_{h}^{*}\right)\right)^{2}}{N_{h}}-\frac{\left(d_{h}+\mu_{h}\right)\left(I_{h}-I_{h}^{*}\right)^{2}}{N_{h}} \\
& +\frac{N_{h}^{*}\left(d_{h}+2 \mu_{h}\right)}{\beta_{h}\left(I_{h}^{*}+R_{h}^{*}\right)}\left(\frac{\beta_{a_{2}} I_{a}^{*}\left(I_{h}-I_{h}^{*}\right)\left(S_{h}-S_{h}^{*}\right)}{N_{a}^{*} I_{h}^{*}}\right) \\
& -\left(\frac{N_{h}^{*}\left(d_{h}+2 \mu_{h}\right)}{\beta_{h}\left(I_{h}^{*}+R_{h}^{*}\right)}\right)\left(\frac{\beta_{a_{2}} I_{a}^{*} S_{h}\left(I_{h}-I_{h}^{*}\right)^{2}}{N_{a}^{*} I_{h} I_{h}^{*}}+\frac{\beta_{h} S_{h}^{*}\left(I_{h}-I_{h}^{*}\right)^{2}}{N_{h}^{*} N_{h}}\right) \\
& -\frac{\left(d_{h}+2 \mu_{h}\right)}{2 \rho_{h}}\left(1+\frac{S_{h}^{*}}{I_{h}^{*}+R_{h}^{*}}\right)\left(\frac{2 \mu_{h}\left(R_{h}-R_{h}^{*}\right)^{2}}{N_{h}}+\frac{N_{h}^{\prime}\left(R_{h}-R_{h}^{*}\right)^{2}}{N_{h}^{2}}\right) \\
& -2 D \mu_{h}\left(\left(S_{h}-S_{h}^{*}\right)-\left(R_{h}-R_{h}^{*}\right)\right)^{2}-2 D\left(d_{h}+2 \mu_{h}\right)\left(I_{h}-I_{h}^{*}\right)^{2} \\
& -2 D\left(d_{h}+2 \mu_{h}\right)\left(S_{h}-S_{h}^{*}\right)\left(I_{h}-I_{h}^{*}\right)-2 D\left(d_{h}+2 \mu_{h}\right)\left(I_{h}-I_{h}^{*}\right)\left(R_{h}-R_{h}^{*}\right) \\
& +2 E \rho_{h}\left(I_{h}-I_{h}^{*}\right)\left(R_{h}-R_{h}^{*}\right)-2 E \mu_{h}\left(R_{h}-R_{h}^{*}\right)^{2} .
\end{aligned}
$$

At this point we can see that most terms of $L^{\prime}$ are negative semi-definite in $\Omega_{h}$, except for the third term, some of the ones involving parameters $D$ and $E$, and potentially the fifth term involving $N_{h}^{\prime}$. To deal with this term we utilize the assumption that $\mu_{h}>d_{h}$, and 
obtain

$$
\begin{gathered}
\frac{2 \mu_{h}\left(R_{h}-R_{h}^{*}\right)^{2}}{N_{h}}+\frac{N_{h}^{\prime}\left(R_{h}-R_{h}^{*}\right)^{2}}{N_{h}^{2}}=\frac{\left(2 \mu_{h} N_{h}+N_{h}^{\prime}\right)\left(R_{h}-R_{h}^{*}\right)^{2}}{N_{h}^{2}} \\
=\frac{\left(2 \mu_{h}\left(S_{h}+I_{h}+R_{h}\right)+\Lambda_{h}-\mu_{h}\left(S_{h}+I_{h}+R_{h}\right)-d_{h} I_{h}\right)\left(R_{h}-R_{h}^{*}\right)^{2}}{N_{h}^{2}} \\
=\frac{\left(\mu_{h}\left(S_{h}+R_{h}\right)+\Lambda_{h}+\left(\mu_{h}-d_{h}\right) I_{h}\right)\left(R_{h}-R_{h}^{*}\right)^{2}}{N_{h}^{2}} \geq 0 .
\end{gathered}
$$

Now we can choose the values of $D$ and $E$ in such a way that all the remaining terms that may not be negative semi-definite cancel out. In particular, let

$$
\begin{aligned}
& \frac{N_{h}^{*}\left(d_{h}+2 \mu_{h}\right)}{\beta_{h}\left(I_{h}^{*}+R_{h}^{*}\right)}\left(\frac{\beta_{a_{2}} I_{a}^{*}}{N_{a}^{*} I_{h}^{*}}\right)-2 D\left(d_{h}+2 \mu_{h}\right)=0 \quad \text { and } \\
& \quad-2 D\left(d_{h}+2 \mu_{h}\right)+2 E \rho_{h}=0 .
\end{aligned}
$$

This implies

$$
\begin{aligned}
& D=\frac{N_{h}^{*} \beta_{a_{2}} I_{a}^{*}}{2 \beta_{h} N_{a}^{*} I_{h}^{*}\left(I_{h}^{*}+R_{h}^{*}\right)}>0 \text { and } \\
& E=\frac{N_{h}^{*} \beta_{a_{2}} I_{a}^{*}\left(d_{h}+2 \mu_{h}\right)}{2 \rho_{h} \beta_{h} N_{a}^{*} I_{h}^{*}\left(I_{h}^{*}+R_{h}^{*}\right)}>0,
\end{aligned}
$$

and with these parameters $L^{\prime}$ is negative semi-definite in $\Omega_{h}$, with $L^{\prime}=0$ if and only if $S_{h}=$ $S_{h}^{*}, I_{h}=I_{h}^{*}, R_{h}=R_{h}^{*}$. Thus the largest compact invariant set in $\left\{\left(S_{h}, I_{h}, R_{h}\right) \in \Omega_{h}: L^{\prime}=0\right\}$ is $\left\{E_{h}^{*}\right\}$, therefore, by the LaSalle invariance principle $\left\{E_{h}^{*}\right\}$ is globally asymptotically stable in $\Omega_{h}$ (LaSalle, 1976; LaSalle \& Lefschetz, 1961). Now Corollary 3.3 implies that $\mathcal{E}_{2}$ is a globally asymptotically stable equilibrium of (1a)-(1f).

Note that our theorem shows that if the disease is endemic in the reservoir population, and $\beta_{a_{2}}>0$, then irrespective of the reproductive number $\mathcal{R}_{0_{h}}$ the disease becomes endemic in the human population (if $\mu_{h}>d_{h}$ ).

\subsection{Disease-free equilibrium in the reservoir population}

The other equilibrium of the reservoir system (1a)-(1c) is the disease-free equilibrium given by $E_{a}=\left(S_{a}^{0}, I_{a}^{0}, R_{a}^{0}\right)=\left(\Lambda_{a} / \mu_{a}, 0,0\right)$. By Theorem 3.1 we know that this equilibrium is globally asymptotically stable if $\mathcal{R}_{0_{a}} \leq 1$. In this case, $I_{a}(t) \rightarrow 0$ as $t \rightarrow \infty$, so in the limit there is no infection in population $H$ coming from the reservoir population $A$. Thus, in the limit, dynamics in population $H$ become exactly the same as the general dynamics in the reservoir population, and Corollary 3.3 applies. Thus we have the following results.

Proposition 3.6: Assume that $\mathcal{R}_{0_{a}} \leq 1$, so the disease dies out in the reservoir population. Additionally, let $\mathcal{R}_{0_{h}} \leq 1$. Then the disease-free equilibrium $\mathcal{E}_{0}=\left(\Lambda_{a} / \mu_{a}, 0,0, \Lambda_{h} / \mu_{h}, 0,0\right)$ of (1a)-(1f) is globally asymptotically stable in $\Omega$. 
Proposition 3.7: Assume that $\mathcal{R}_{0_{a}} \leq 1, \mathcal{R}_{0_{h}}>1$ and $\mu_{h}>d_{h}$. Then the equilibrium $\mathcal{E}_{2}=\left(\Lambda_{a} / \mu_{a}, 0,0, S_{h}^{*}, I_{h}^{*}, R_{h}^{*}\right)$ of (1a)-(1f) is globally asymptotically stable in $\Omega$. (Note that $\left(S_{h}^{*}, I_{h}^{*}, R_{h}^{*}\right)$ are given by the same expressions as $(2 \mathrm{a})-(2 \mathrm{c})$ with the parameters corresponding to population $H$.)

\section{Numerical results and possible modifications to the model}

In this section we present some numerical results related to the model (1a)-(1f) with animals as the reservoir population $A$ and humans represented as population $H$. Figure 1 shows the results of a numerical simulation of (1a)-(1f) using MATLAB's ode 45 and the parameters $\Lambda_{a}=152500 / 3, \mu_{a}=1 / 8, \rho_{a}=1 / 20, d_{a}=1 / 30, \Lambda_{h}=2900 / 6, \mu_{h}=$
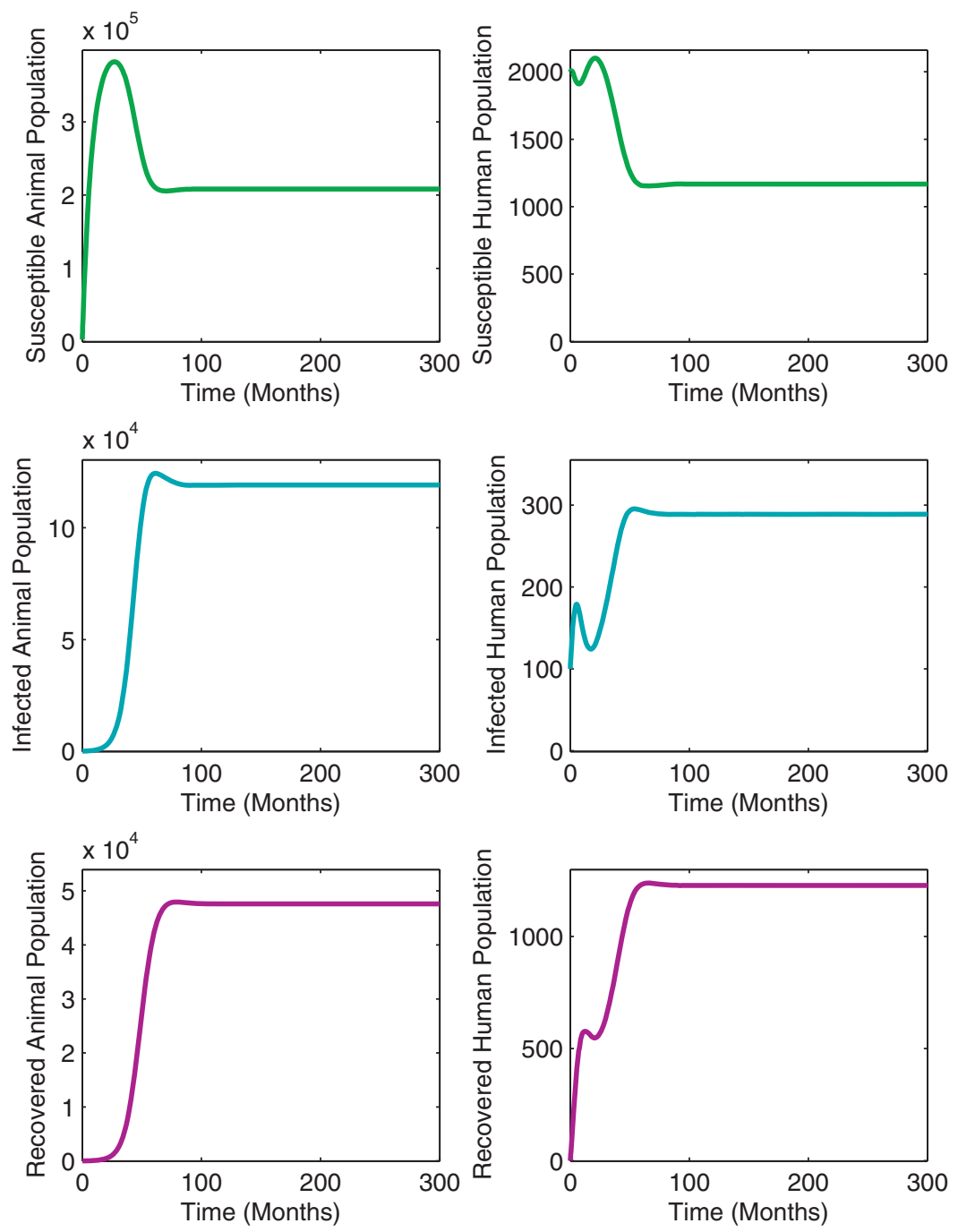

Figure 1. The above shows the results for a simulation with all parameters constant and the criteria for Theorem 3.5 met. 
$1 / 6, \rho_{h}=17 / 24, d_{h}=1 / 8$ individuals per month and $\beta_{h}=31 / 24, \beta_{a_{1}}=3 / 8$ and $\beta_{a_{2}}=$ $41 / 120$ as the contact rates. The initial values used were $S_{h}^{0}=2000, I_{h}^{0}=R_{h}^{h}=R_{a}^{0}=$ $0, S_{a}^{0}=30000$ and $I_{a}^{0}=1000$. These are artificial values and are used only for illustration purposes. Under these conditions, the endemic equilibrium of (1a)-(1f) is globally asymptotically stable.

We used monkeypox as a motivating example for our next simulation. Since there is no longer any vaccination against smallpox and this vaccine provided some immunity against monkeypox, there is waning herd immunity against monkeypox (Hutin et al., 2001; Levine et al., 2007; Lloyd-Smith et al., 2009; McCollum \& Damon, 2014; Nolen et al., 2016; Rimoin \& Graham, 2011; Rimoin et al., 2007, 2010). Thus, for the next simulation, we assume that as time goes on there is an increasing likelihood that a human will get infected when they come into contact with an infected animal. For this simulation, we use $\beta_{a_{2}}(t)=1 /\left(1+9 \mathrm{e}^{-t}\right)$. Figure 2 shows the results of a numerical simulation with this
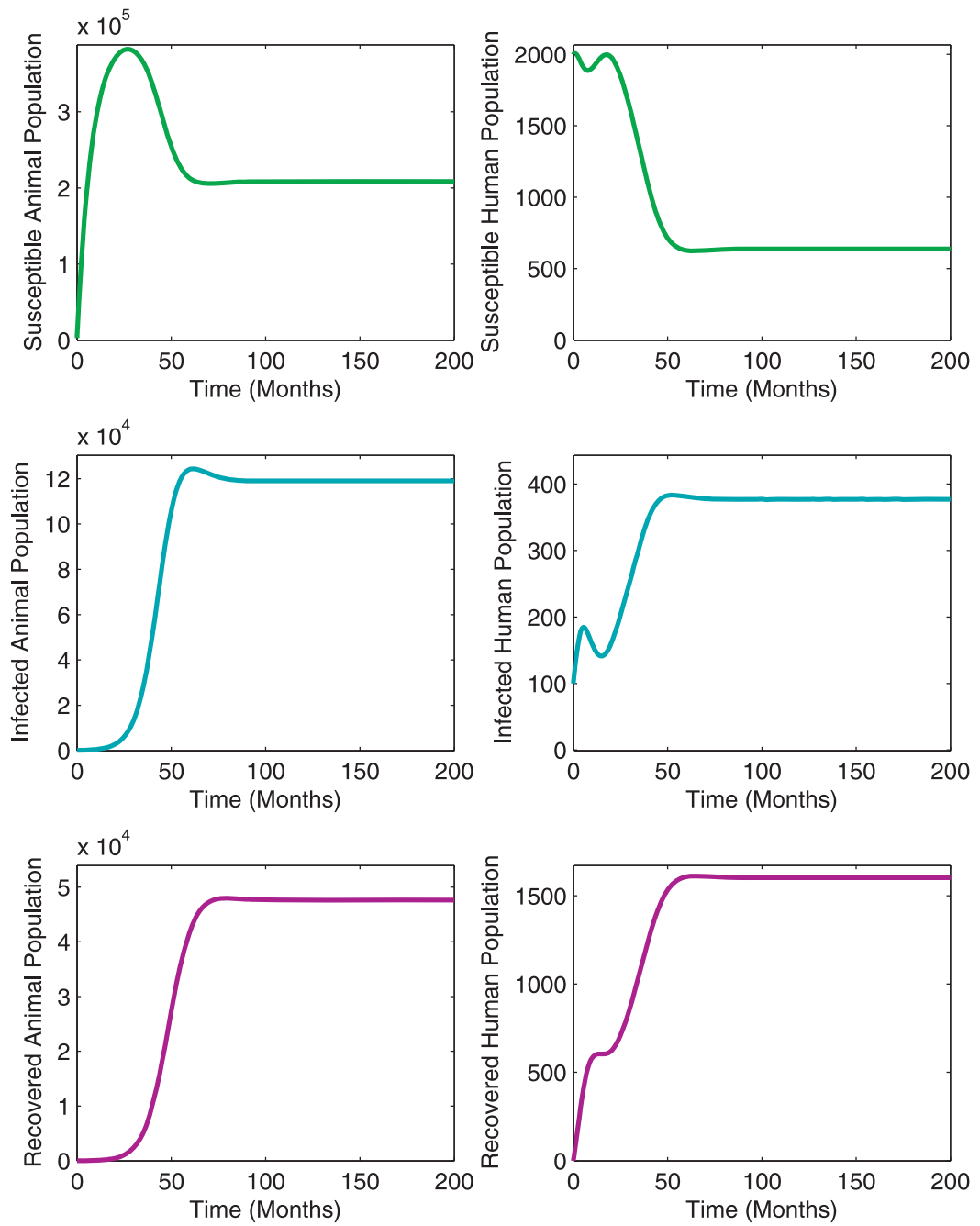

Figure 2. The results for a simulation with all parameters constant except $\beta_{a_{2}}(t)$ which is an increasing function of time. 
$\beta_{a_{2}}(t)$ and all other parameters the same as for Figure 1 . The analysis for our asymptotically autonomous system is still valid because $\beta_{a_{2}}(t) \rightarrow 1$ as $t \rightarrow \infty$ and so if we define $g(t):=\beta_{a_{2}}(t) I_{a}(t) / N_{a}(t)$, then $g(t) \rightarrow I_{a}^{e} / N_{a}^{e}$ as $t \rightarrow \infty$. Since $\beta_{a_{2}} \rightarrow 1$, it makes sense that Figure 2 shows the system approaching a higher $I_{h}^{*}$ value than in Figure 1.

In the proof of the global asymptotic stability for the co-existence equilibrium, we assumed that $\mu_{a}>d_{a}$ and $\mu_{h}>d_{h}$. However, even if we break both of those conditions, the numerical results still seem to indicate that the co-existence endemic equilibrium of (1a)-(1f) is globally asymptotically stable. Figure 3 shows the results of a simulation of this kind, with $\Lambda_{a}=152500 / 3, \mu_{a}=1 / 25, \rho_{a}=1 / 20, d_{a}=1 / 10, \Lambda_{h}=2900 / 6, \mu_{h}=$ $1 / 9, \rho_{h}=17 / 24, d_{h}=1 / 6$ individuals per month and $\beta_{h}=31 / 24, \beta_{a_{1}}=3 / 8$ and $\beta_{a_{2}}=$
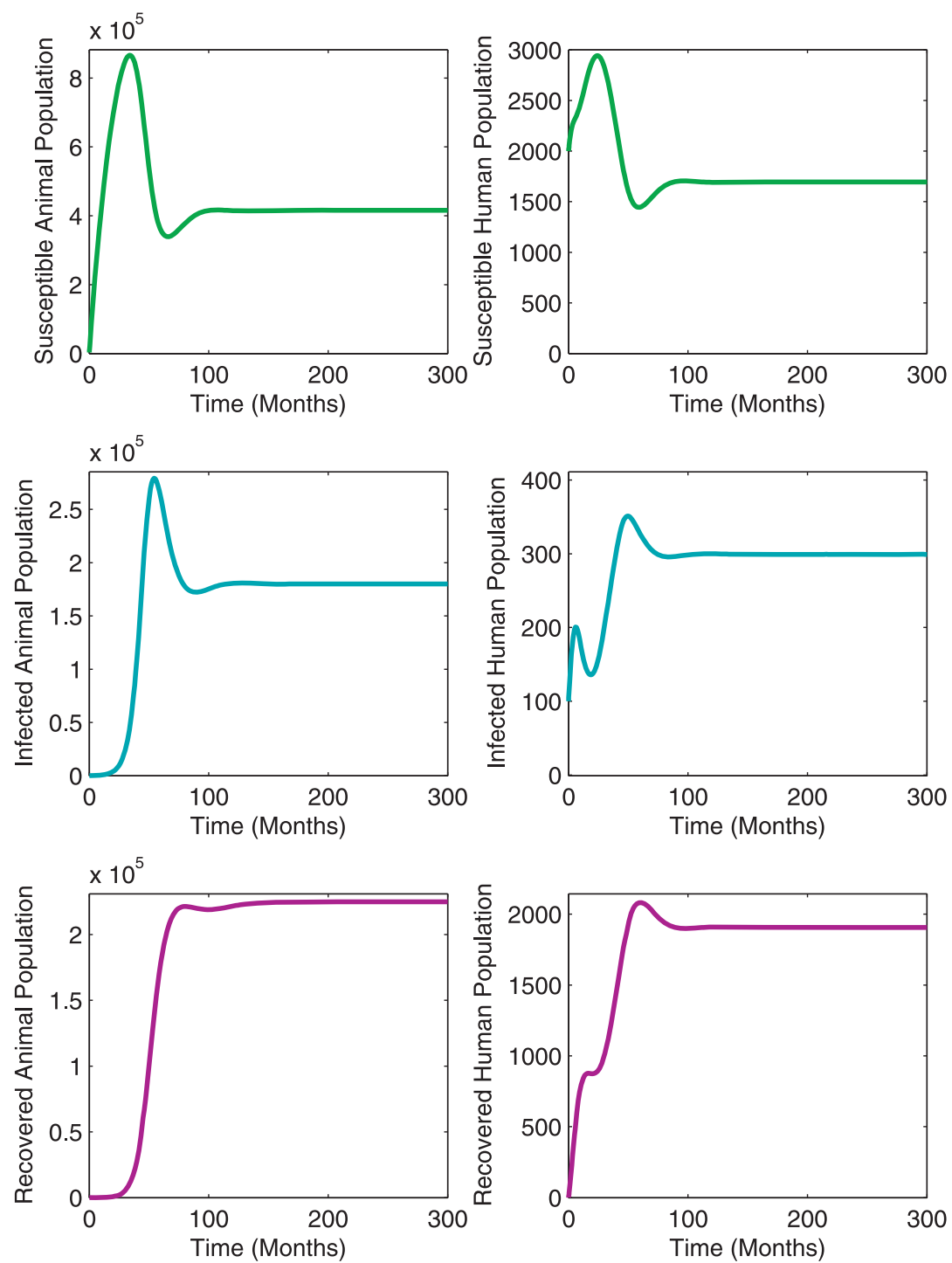

Figure 3. The results of a simulation with all parameters constant, but with $\mu_{a}<d_{a}$ and $\mu_{h}<d_{h}$. 
$41 / 120$ as the contact rates. The initial values used are the same as those in the previous two sets of results.

We also simulate a more complicated scenario where there are multiple reservoir populations. Suppose there are $n$ such reservoir populations, $A_{1}, A_{2}, \ldots, A_{n}$ and one population $H$ as in the previous model. $S_{h}, I_{h}, R_{h}$ and $N_{h}$ are defined as before with $S_{a_{i}}, I_{a_{i}}$ and $R_{a_{i}}$ representing the susceptible, infected and recovered individuals in population $A_{i}$, for $i=1,2, \ldots, n$, with the total number of individuals in population $A_{i}$ being given as $N_{a_{i}}$. Susceptible individuals in $A_{i}$ are recruited through migration and birth at the rate $\Lambda_{a_{i}}$ and susceptible individuals in $H$ are recruited at a rate of $\Lambda_{h}$. We represent the death rates from disease in population $A_{i}$ by $d_{a_{i}}$ and the death rate by disease in population $H$ as $d_{h}$. Further, we assume $\mu_{a_{i}}, \mu_{h}$ are the natural death rates for $A_{i}$ and $H$, respectively, and $\rho_{a_{i}}, \rho_{h}$ be the recovery rates with permanent immunity for $A_{i}$ and $H$, respectively. It is assumed that no one in population $H$ can infect any individual in any $A_{i}$ population, while individuals in any $A_{i}$ population can infect those in $H$ on suitable contact. We assume there is no crossinfection between $A_{i}$ and $A_{j}$ when $i \neq j$. Disease transmission is modelled using standard incidence, assuming a constant (density-independent) contact rate both within and across the two populations resulting in infection rates

$$
\begin{aligned}
& f_{a_{i}}\left(S_{a_{i}}, I_{a_{i}}, R_{a_{i}}\right)=\frac{\beta_{a_{i 1}} I_{a_{i}}}{N_{a_{i}}} S_{a_{i}}, \quad \text { for } i=1,2, \ldots, n, \text { and } \\
& f_{h}\left(S_{a_{1}}, I_{a_{1}}, R_{a_{1}}, \ldots, S_{a_{n}}, I_{a_{n}}, R_{a_{n}}, S_{h}, I_{h}, R_{h}\right)=\left(\left(\sum_{i=1}^{n} \frac{\beta_{a_{i 2}} I_{a_{i}}}{N_{a_{i}}}\right)+\frac{\beta_{h} I_{h}}{N_{h}}\right) S_{h},
\end{aligned}
$$

where $\beta_{a_{i 1}}$ is the effective contact rate within population $A_{i}, \beta_{a_{i 2}}$ is the effective contact rate between populations $A_{i}$ and $H$ and $\beta_{h}$ is the effective contact rate within population $H$. We assume that, for $i=1,2, \ldots, n, \Lambda_{a_{i}}, \Lambda_{h}, \mu_{a_{i}}, \mu_{h}, \rho_{a_{i}}, \rho_{h}$ are positive parameters and $d_{a_{i}}, d_{h}, \beta_{a_{1 i}}, \beta_{a_{2 i}}$ and $\beta_{h}$ are non-negative parameters. Specifically, this leads to the following model. For $i=1,2, \ldots, n$,

$$
\begin{aligned}
& \frac{\mathrm{d} S_{a_{i}}}{\mathrm{~d} t}=\Lambda_{a_{i}}-\mu_{a_{i}} S_{a_{i}}-\frac{\beta_{a_{i 1}} I_{a_{i}}}{N_{a_{i}}} S_{a_{i}}, \\
& \frac{\mathrm{d} I_{a_{i}}}{\mathrm{~d} t}=\frac{\beta_{a_{1 i}} I_{a_{i}}}{N_{a_{i}}} S_{a_{i}}-\left(\mu_{a_{i}}+\rho_{a_{i}}+d_{a_{i}}\right) I_{a_{i}}, \\
& \frac{\mathrm{d} R_{a_{i}}}{\mathrm{~d} t}=\rho_{a_{i}} I_{a_{i}}-\mu_{a_{i}} R_{a_{i}}, \\
& \frac{\mathrm{d} S_{h}}{\mathrm{~d} t}=\Lambda_{h}-\mu_{h} S_{h}-\left(\left(\sum_{i=1}^{n} \frac{\beta_{a_{i 2}} I_{a_{i}}}{N_{a_{i}}}\right)+\frac{\beta_{h} I_{h}}{N_{h}}\right) S_{h}, \\
& \frac{\mathrm{d} I_{h}}{\mathrm{~d} t}=\left(\left(\sum_{i=1}^{n} \frac{\beta_{a_{i 2}} I_{a_{i}}}{N_{a_{i}}}\right)+\frac{\beta_{h} I_{h}}{N_{h}}\right) S_{h}-\left(\mu_{h}+\rho_{h}+d_{h}\right) I_{h},
\end{aligned}
$$




$$
\frac{\mathrm{d} R_{h}}{\mathrm{~d} t}=\rho_{h} I_{h}-\mu_{h} R_{h}
$$

Let

$$
\Omega_{a_{i}}=\left\{\left(S_{a_{i}}, I_{a_{i}}, R_{a_{i}}\right) \in \mathbb{R}_{+}^{3}: S_{a_{i}} \geq 0, I_{a_{i}} \geq 0, R_{a_{i}} \geq 0, S_{a_{i}}+I_{a_{i}}+R_{a_{i}} \leq \frac{\Lambda_{a_{i}}}{\mu_{a_{i}}}\right\}
$$

and

$$
\Omega_{h}=\left\{\left(S_{h}, I_{h}, R_{h}\right) \in \mathbb{R}_{+}^{3}: S_{h} \geq 0, I_{h} \geq 0, R_{h} \geq 0, S_{h}+I_{h}+R_{h} \leq \frac{\Lambda_{h}}{\mu_{h}}\right\} .
$$

Then for $i=1,2, \ldots, n$, the set $\tilde{\Omega}=\Omega_{a_{1}} \times \Omega_{a_{2}} \times \cdots \times \Omega_{a_{n}} \times \Omega_{h}$ is positively invariant under the dynamics of (14a)-(14f) and solutions with initial conditions in $\tilde{\Omega}$ exist globally.

Define $E_{a_{i}}^{(t)}:=\left(S_{a_{i}}^{(t)}, I_{a_{i}}^{(t)}, R_{a_{i}}^{(t)}\right)$ and $E_{a_{i}}^{e}:=\left(S_{a_{i}}^{e}, I_{a_{i}}^{e}, R_{a_{i}}^{e}\right)$ for $i=1,2, \ldots, n$. Depending on the parameters, $E_{a_{i}}^{e}$ is either the disease-free equilibrium or the endemic equilibrium. By the structure of the model in (14a)-(14f) - with animals uninfected by humans and each animal population independent, that is, unable to infect any other animal population - we have that $E_{a_{i}}^{(t)} \rightarrow E_{a_{i}}^{e}$ for $i=1,2, \ldots, n$, and each $E_{a_{i}}^{e}$ is globally asymptotically stable by Theorems 3.1 and 3.2 under appropriate conditions. Thus, it is straightforward to extend the results of the equilibrium analysis for (1a)-(1f) and we have the following corollary.

Corollary 4.1: If there exists an $i \in 1,2, \ldots, n$ such that $\mathcal{R}_{0_{a_{i}}}>1, \mu_{a_{i}}>d_{a_{i}}$ and $\beta_{a_{i 2}}>0$, and $\mu_{h}>d_{h}$, then the unique endemic equilibrium $\tilde{\mathcal{E}}=\left(E_{a_{1}}^{e}, \ldots, E_{a_{n}}^{e}, E_{h}^{*}\right)$ of $(14 \mathrm{a})-(14 \mathrm{f})$ is globally asymptotically stable in the interior of $\tilde{\Omega}$.

In the simplest scenario for this model, we assume $i=2$. Figure 4 shows the results of an epidemic simulation with two animal populations and one human population. The initial values used for this simulation are $S_{a_{1}}^{0}=3000, I_{a_{1}}^{0}=100, S_{a_{2}}^{0}=2500, I_{a_{2}}^{0}=40, S_{h}^{0}=$ 2000, $I_{h}^{0}=100$ and $R_{a_{1}}^{0}=R_{a_{2}}^{0}=R_{h}^{0}=0$. The contact rates are $\beta_{a_{11}}=1 / 4, \beta_{a_{21}}=$ $1 / 9, \quad \beta_{a_{12}}=1 / 8, \quad \beta_{a_{22}}=1 / 11$ and $\beta_{h}=31 / 24$. The parameter values, with units of individuals per month, are $\Lambda_{h}=2900 / 6, \mu_{h}=1 / 6, \rho_{h}=17 / 24, d_{h}=1 / 8, \Lambda_{a_{1}}=$ $152500 / 2, \mu_{a_{1}}=1 / 8, \rho_{a_{1}}=1 / 20, d_{a_{1}}=1 / 30, \Lambda_{a_{2}}=500, \mu_{a_{2}}=1 / 25, \rho_{a_{2}}=1 / 10$ and $d_{a_{2}}=1 / 30$.

Looking at the system (1a)-(1f) and revisiting our assumptions, the infection in the human population depends on infection in both populations. In Figure 5 we see the change in $I_{h}^{*} / N_{h}^{*}$ as $\mathcal{R}_{0_{a}}$ changes. The values used for this figure were $\Lambda_{a}=152500 / 3, \mu_{a}=$ $1 / 25, \rho_{a}=1 / 20, d_{a}=1 / 10, \Lambda_{h}=2900 / 6, \mu_{h}=1 / 9, \rho_{h}=17 / 24, d_{h}=1 / 6$ individuals per month and $\beta_{a_{2}}=0.3$. In order to get the change in $\mathcal{R}_{0_{a}}$, we use a range of $\beta_{a_{1}}$ values, namely $0 \leq \beta_{a_{1}} \leq 1.9$. The initial values used were $S_{h}^{0}=2000, I_{h}^{0}=R_{h}^{h}=R_{a}^{0}=0, S_{a}^{0}=$ 3000 and $I_{a}^{0}=1000$. (These values are simply for illustration purposes.) We notice that $I_{h}^{*} / N_{h}^{*}$ increases after $\mathcal{R}_{0_{a}}=1$. When $\beta_{h}=1 / 3$ we have $\mathcal{R}_{0_{h}} \approx 0.338$ and when $\beta_{h}=1 / 9$ we have $\mathcal{R}_{0_{h}} \approx 0.113$. Even if $\beta_{h}=0$, when $\mathcal{R}_{0_{a}} \geq 1$, we see there is infection in the human population so limiting infection from humans to humans is not enough to fully mitigate this disease in humans. In all three curves there is a sharp increase in $I_{h}^{*} / N_{h}^{*}$ for $1 \leq \mathcal{R}_{0_{a}} \leq 2$ so that the differences between the different $I_{h}^{*} / N_{h}^{*}$ curves are nearly indistinguishable the closer $\mathcal{R}_{0_{a}}$ is to 1 . While we do not know what $\beta_{a_{1}}$ is in reality, this shows 

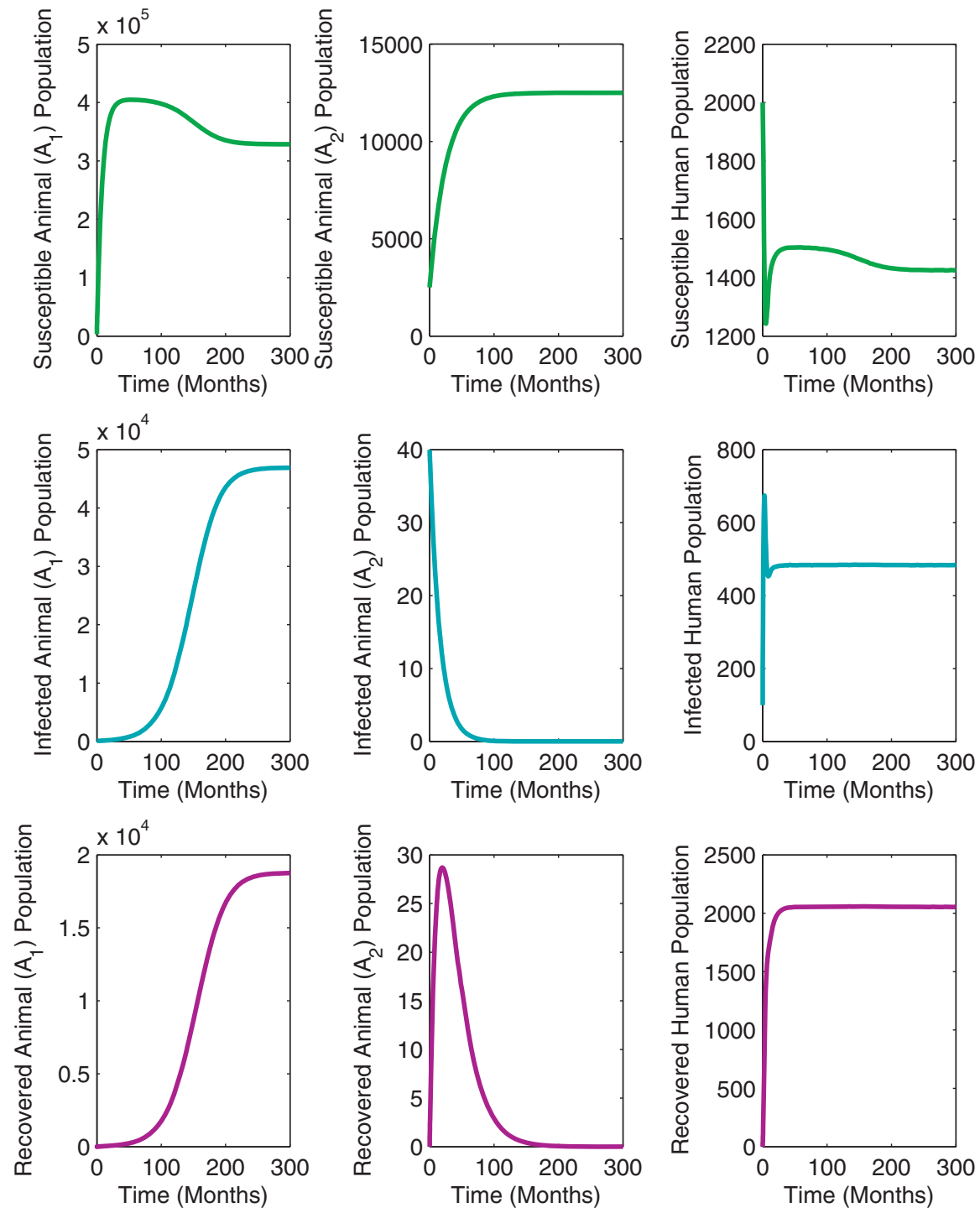

Figure 4. The results of a simulation with two animal populations as in (14a)-(14f) for $i=2$. The first two columns show the results for populations $A_{1}$ and $A_{2}$, respectively. The last column shows the results for the human population.

that if it is high enough for there to be endemic infection in the animal population, there will be some level of infection in the human population. Figure 6 uses the same parameters as in 5 , but with $\beta_{h} \geq 1$. Specifically, $\beta_{h}=1$ where $\mathcal{R}_{0_{h}} \approx 1.014, \beta_{h}=1.5$ when we have $\mathcal{R}_{0_{h}} \approx 1.521$ and $\beta_{h}=2$ when $\mathcal{R}_{0_{h}} \approx 2.028$. As expected, the increase in $\beta_{h}$ results in higher values for $I_{h}^{*} / N_{h}^{*}$ than in Figure 5. While difficult, it is important to continue studying monkeypox in both human and animal populations since infection in the animal population has a substantial impact on infection in the human population.

In Figure 7, we use the same parameters as in Figure 5, but we consider $I_{a}^{*} / N_{a}^{*}$ as a function of $\mathcal{R}_{0_{a}}$. Since we changed $\beta_{h}$ to obtain three curves in Figure 5 but $\beta_{h}$ has no 


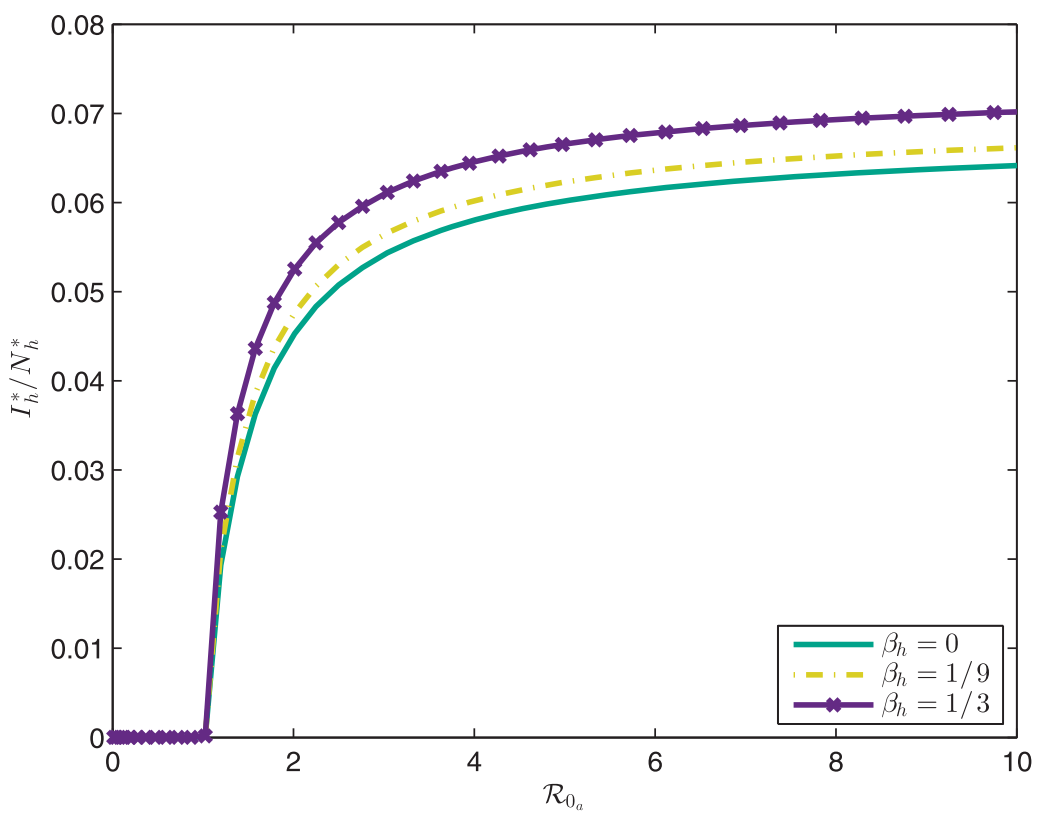

Figure 5. The above illustrates the ratio $l_{h}^{*} / N_{h}^{*}$ as a function of $\mathcal{R}_{0_{a}}$. The lowest curve represents the case when $\beta_{h}=0$ so we see that there is still infection in the human population as long as $\mathcal{R}_{0_{a}} \geq 1$.

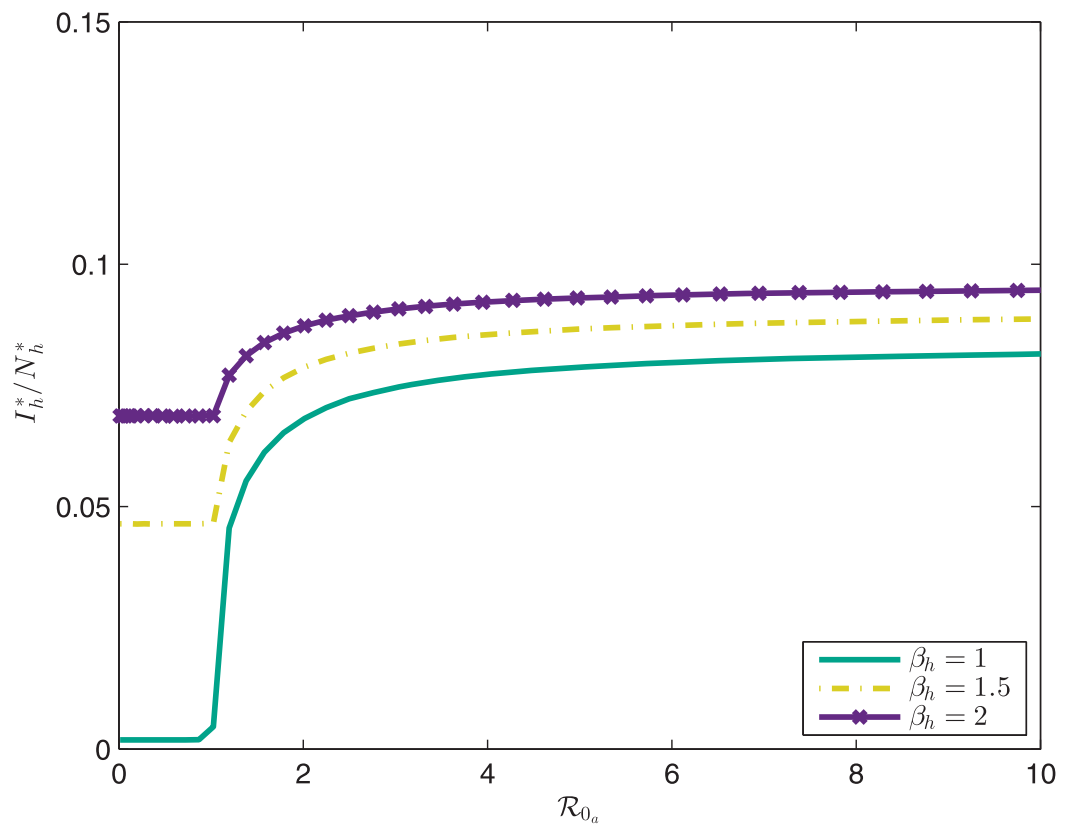

Figure 6. The above illustrates the ratio $l_{h}^{*} / N_{h}^{*}$ as a function of $\mathcal{R}_{0_{a}}$ for $\mathcal{R}_{0_{h}}>1$.

role in the value of $I_{a}^{*} / N_{a}^{*}$, the curve in Figure 7 is the same regardless of $\beta_{h}$. We note that, as would be expected, changing $\mathcal{R}_{0_{a}}$ has a greater effect on $I_{a}^{*} / N_{a}^{*}$ than it did on $I_{h}^{*} / N_{h}^{*}$ reflected in Figure 5. 


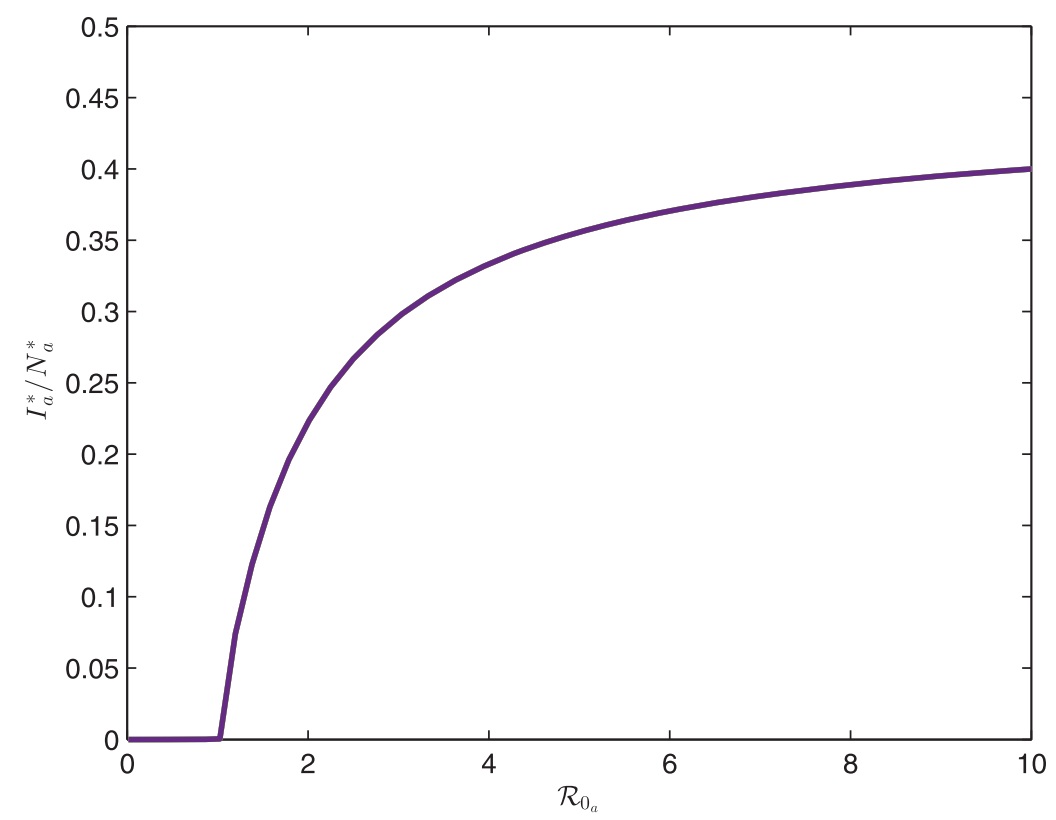

Figure 7. The above illustrates $I_{a}^{*} / N_{a}^{*}$ as a function of $\mathcal{R}_{0_{a}}$.

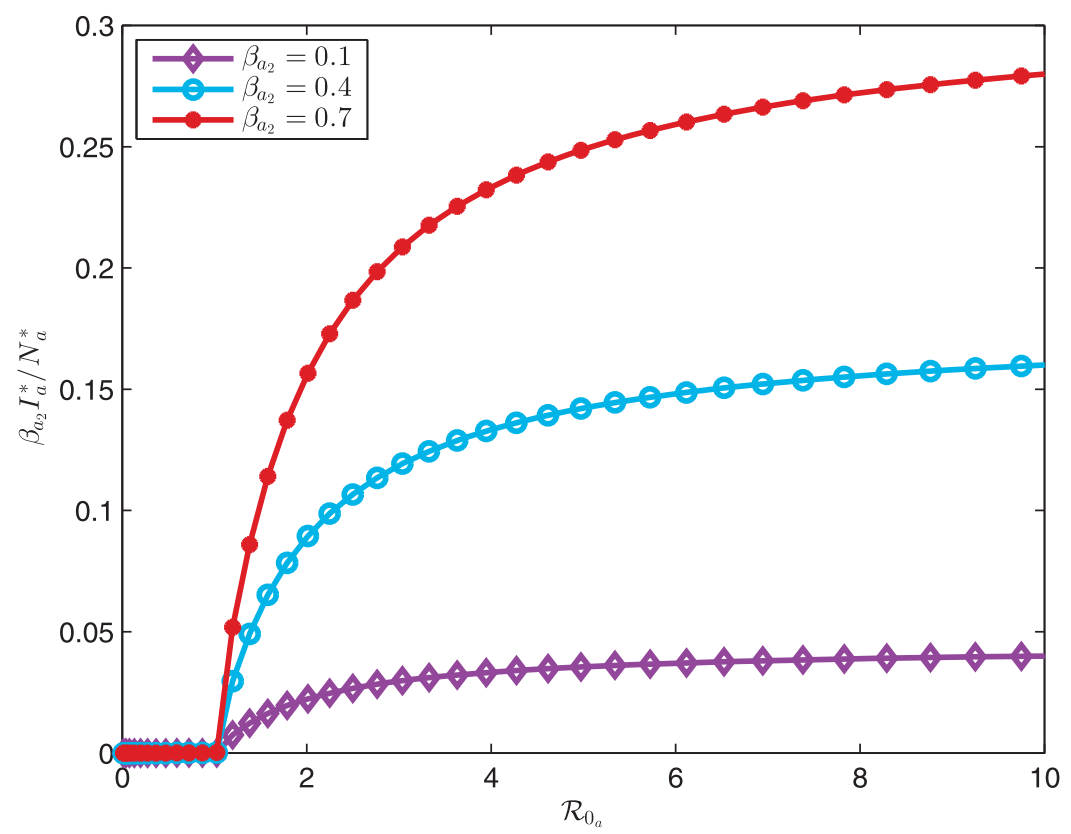

Figure 8. The above illustrates $\beta_{a_{2}} l_{a}^{*} / N_{a}^{*}$ as a function of $\mathcal{R}_{0_{a}}$ for three different values of $\beta_{a_{2}}$ with $\beta_{h}=0$.

We also know that changes in $\beta_{a_{2}}$ impact the animal-to-human cross-infection. Figure 8 shows $\beta_{a_{2}} I_{a}^{*} / N_{a}^{*}$ as a function of $\mathcal{R}_{0_{a}}$ for the same values as in Figure 5 with $\beta_{h}=0$. These results indicate that controlling the disease in the human population also depends on reducing the value of $\beta_{a_{2}}$, as would be expected. Continuing to educate people in areas 
affected by monkeypox on recognizing the symptoms and proper handling of infected animals is crucial in limiting the spread of this disease among humans.

Future modifications to the model include possibly making the migration and birth rates functions of time, $\Lambda_{a}(t)$ and $\Lambda_{h}(t)$, in addition to experimenting with different functions for $\beta_{a_{2}}(t)$. These adjustments may more accurately convey seasonal influences as well as other environmental influences and trends.

\section{Acknowledgments}

The authors wish to thank the anonymous reviewers for their helpful input.

\section{Disclosure statement}

No potential conflict of interest was reported by the authors.

\section{References}

Afassinou, K., Chirove, F., \& Govinder, K. S. (2017). Pre-exposure prophylaxis and antiretroviral treatment interventions with drug resistance. Mathematical Biosciences, 285, 92-101.

Bhunu, C. P., \& Mushayabasa, C. (2011). Modelling the transmission dynamics of pox-like infections. IAENG International Journal of Applied Mathematics, 41(2), 141-149.

Bhunu, C. P., Mushayabasa, C., \& Hyman, J. M. (2012). Modelling HIV/AIDS and monkeypox coinfection. Applied Mathematics and Computation, 218, 9504-9518.

Castillo-Chavez, C., Feng, Z., \& Huang, W. (2001). On the computation of $\mathcal{R}_{0}$ and its role on global stability. Institute for Mathematics and Its Applications, 125, 229.

Castillo-Chavez, C., \& Thieme, H. (1994). Asymptotically autonomous epidemic models. Biometrics Unit Technical Reports, BU-1248-M. Retrieved from http://hdl.handle.net/1813/31834

Centers for Disease Control and Prevention (2015, May 11). Monkeypox. Retrieved from https://www.cdc.gov/poxvirus/monkeypox/

Centers for Disease Control and Prevention (2017a, April 17). Information on Avian Influenza. Retrieved from https://www.cdc.gov/flu/avianflu/index.htm

Centers for Disease Control and Prevention (2017b, December 27). About Ebola Virus Disease. Retrieved from https://www.cdc.gov/vhf/ebola/about.html

Damon, I. K. (2011). Status of human monkeypox: Clinical disease, epidemiology and research. Vaccine, 29(Suppl 4), D54-D59. doi:10.1016/j.vaccine.2011.04.014

Gilbert, A. T., McCracken, G. F., Sheeler, L. L., Muller, L. I., O’Rourke, D., Kelch, W. J., \& New, J. C., Jr. (2015). Rabies surveillance among bats in Tennessee, USA, 1996-2010. Journal of Wildlife Diseases, 51(4), 821-832. doi:10.7589/2014-12-277

Hammarlund, E., Lewis, M. W., Carter, S. V., Amanna, I., Hansen, S. G., Strelow, L. I., . . Silfka, M. K. (2005). Multiple diagnostic techniques identify previously vaccinated individuals with protective immunity against monkeypox. Nature Medicine, 11(9), 1005-1011.

Hutin, Y. J., Williams, R. J., Malfait, P., Pebody, R., Loparev, V. N., Ropp, S. L., ... Esposito, J. J. (2001). Outbreak of human monkeypox, Democratic Republic of Congo, 1996-1997. Emerging Infectious Diseases, 7, 434-438. doi:10.3201/eid0703.010311

Jezek, Z., Arita, I., Mutombo, M. D., Nakano, J. H., \& Szczeniowski, M. (1986). Four generations of probable person-to-person transmission of human monkeypox. American Journal of Epidemiology, 123(6), 1004-1012.

Jezek, Z., Grab, B., Szczeniowski, M. V., Paluku, K. M., \& Mutombo, M. (1988). Human monkeypox: Secondary attack rates. Bulletin of the World Health Organization, 66(4), 465-470.

Kantele, A., Chickering, K., Vapalahti, Q., \& Rimoin, A. W. (2016). Emerging diseases - the monkeypox epidemic in the democratic republic of the Congo. Clinical Microbiology and Infection, 22, 658-659. doi:10.1016/j.cmi.2016.07.004

LaSalle, J. P. (1976). The stability of dynamical systems. Philadelphia, PA: SIAM. 
LaSalle, J. P., \& Lefschetz, S. (1961). Stability by Liapunovs direct method with applications. New York: Academic Press.

Levine, R. S., Townsend Peterson, A., Yorita, K. L., Carroll, D., Damon, I. K., \& Reynolds, M. G. (2007). Ecological niche and geographic distribution of human monkeypox in Africa. PloS One, 2(1), e176. doi:10.1371/journal.pone.0000176

Lloyd-Smith, J. O., George, D., Pepin, K. M., Pitzer, V. E., Pulliam, J. R., Dobson, A. P., .. Grenfell, B. T. (2009). Epidemic dynamics at the human-animal interface. Science, 326, 1362-1367.

Markus, L. (1956). Asymptotically autonomous differential systems. In S. Lefschetz (Ed.), Annals of mathematics studies: Vol. 36. Contributions to the theory of nonlinear oscillations III. London: Princeton University Press.

McCollum, A. M., \& Damon, I. K. (2014). Human monkeypox. Clinical Infectious Diseases, 58, 260-267. doi:10.1093/cid/cit703

Nolen, L. D., Osadebe, L., Katomba, J., Likofata, J., Mukadi, D., Monroe, B., ... Reynolds, M. G. (2016). Extended human-to-human transmission during a monkeypox outbreak in the Democratic Republic of the Congo. Emerging Infectious Diseases, 22, 1014-1021.

Reynolds, M. G., Emerson, G. L., Pukuta, E., Karhemere, S., Muyembe, J. J., Bikindou, A., ... Mombouli, J. V. (2013). Detection of human monkeypox in the Republic of the Congo following intensive community education. The American Journal of Tropical Diseases and Hygiene, 88(5), 982-985. doi:10.4269/ajtmh.12-0758

Rimoin, A. W., \& Graham, B. S. (2011). Whither monkeypox vaccination. Vaccine, 29(Suppl 4), D60-D64. doi:10.1016/j.vaccine.2011.09.004

Rimoin, A. W., Kisalu, N., Kebela-Ilunga, B., Mukaba, T., Wright, L. L., Formenty, P., ... Meyer, H. (2007). Endemic human monkeypox, Democratic Republic of Congo, 2001-2004. Emerging Infectious Diseases, 13, 934-937. doi:10.3201/eid1306.061540

Rimoin, A. W., Mulembakani, P. M., Johnston, S. C., Lloyd-Smith, J. O., Kisalu, N. K., Kinkela, T. L., .. Muyembe, J. (2010). Major increase in human monkeypox incidence 30 years after smallpox vaccination campaigns cease in the Democratic Republic of Congo. Proceedings of the National Academy of Sciences of the United States of America, 107, 16262-16267. doi:10.1073/pnas.1005769107

The Center for Food Security and Public Health (2013, February). Monkeypox. Retrieved from http://www.cfsph.iastate.edu/Factsheets/pdfs/monkeypox.pdf

Thieme, H. (1992). Convergence results and a Poincaré-Bendixson trichotomy for asymptotically autonomous differential equations. Journal of Mathematical Biology, 30, 755-763.

van den Driessche, P., \& Watmough, J. (2002). Reproduction numbers and sub-threshold endemic equilibria for compartmental models of disease transmission. Mathematical Biosciences, 180, $29-48$.

Vargas-De-León, C. (2011). On the global stability of SIS, SIR and SIRS epidemic models with standard incidence. Chaos Solitons \& Fractals, 44, 1106-1110.

Weaver, J. R., \& Isaacs, S. N. (2009). Monkeypox virus and insights into its immunomodulatory proteins. Immunological Reviews, 225, 96-113.

World Health Organization (2016, November). Monkeypox Fact Sheet. Retrieved from http://www. who.int/mediacentre/factsheets/fs161/en/ 